\title{
A Neural Network-Based Distributed Compressed Sensing Algorithm to Derive Continuous Hyperspectral Images in Wireless Sensor Networks
}

Md. Sarkar Hasanuzzaman ( $\sim$ sr.hzaman@du.ac.bd)

University of Dhaka

\section{Research Article}

Keywords: hyperspectral image, decision support system, super-resolution, deep residual convolutional neural network, residual block

Posted Date: January 10th, 2022

DOI: https://doi.org/10.21203/rs.3.rs-1234545/v1

License: (c) (1) This work is licensed under a Creative Commons Attribution 4.0 International License.

Read Full License 
A Neural network-based Distributed Compressed Sensing algorithm to derive Continuous hyperspectral images in Wireless Sensor Networks

\title{
Md. Sarkar Hasanuzzaman
}

Department of Computer Science and Engineering, Faculty of Engineering and Technology, University of Dhaka, Bangladesh

Email: $\underline{\text { sr.hzaman@du.ac.bd }}$

\begin{abstract}
Hyperspectral imaging is a versatile and powerful technology for gathering geo-data. Planes and satellites equipped with hyperspectral cameras are currently the leading contenders for large-scale imaging projects. Aiming at the shortcomings of traditional methods for detecting sparse representation of multi-spectral images, this paper proposes wireless sensor networks (WSNs) based single-hyperspectral image super-resolution method based on deep residual convolutional neural networks. We propose a different strategy that involves merging cheaper multispectral sensors to achieve hyperspectral-like spectral resolution while maintaining the WSN's spatial resolution. This method studies and mines the nonlinear relationship between low-resolution remote sensing images and high-resolution remote sensing images, constructs a deep residual convolutional neural network, connects multiple residual blocks in series, and removes some unnecessary modules. For this purpose, a decision support system is used that provides the outcome to the next layer. Finally, this paper, fully explores the similarities between natural images and hyperspectral images, use natural image samples to train convolutional neural networks, and further use migration learning to introduce the trained network model to the super-resolution problem of high-resolution remote sensing images, and solve the lack of training samples problem. A comparison between different algorithms for processing data on datasets collected in situ and via remote sensing is used to evaluate the proposed approach. The experimental results show that the method has good performance and can obtain better super-resolution effects.
\end{abstract}

Keywords: hyperspectral image; decision support system; super-resolution; deep residual convolutional neural network; residual block

\section{Introduction}

Hyperspectral images are obtained by imaging the target in the same scene in the ultraviolet, visible, near-infrared, and mid-infrared regions with an imaging spectrometer to form tens or hundreds of spectral bands. It contains not only a wealth of image information, but also spectral information. Some of them are similar to natural images such as target detection, image classification, and image denoising. Also, there are some that are different from natural images such as spectral unmixing. Hyperspectral images have many advantages, but their spatial resolution is low and unreliable in many applications. Therefore, improving the resolution of hyperspectral images is an important scientific research issue. Current super-resolution methods for hyperspectral images can be broadly divided into two categories. The first type is to fuse a low resolution hyperspectral image (HSI) with an auxiliary image (such as a high resolution multispectral image (MSI)) to get a high resolution hyperspectral image. As a classical algorithm, a fusion model based on Bayesian theory plays an important role in 
HSI super-resolution. For example, Akhtar et al. ${ }^{[1]}$ proposed a fusion model of Bayes sparse expression. HSI is estimated by fusing the corresponding MSI. Sparse coding is also an effective HSI super-resolution method and has a wide range of uses for super-resolution of images. For example, Dong et al. ${ }^{[2]}$ first proposed learning a high-resolution dictionary through a low-resolution HSI. Then combine the low resolution HSI to sparsely code the MSI image and get the final estimated high resolution HSI. In addition, regularization methods are also widely used in HSI super-resolution problems, such as Simoes et al. ${ }^{[3]}$ introduced total variation regularity into the HSI fusion problem, and the model constructed effectively improved image resolution. The above method can increase the resolution of HSI to some extent, but all need to rely on the auxiliary multispectral image of the same scene, and the two types of images need to be registered exactly. Meeting these requirements is not easy. In a real application, this classmethod has certain restrictions.

Hyperspectral and multispectral imaging are powerful tools for gathering data about the environment. It's been utilised for a wide range of purposes. Some of the most well-known examples can be seen in agricultural settings ${ }^{[11]}$, where it aids in the evaluation of quality, biomass, crop types, and even the spread of crop diseases. It can also be used in geology to map mineral richness and other things ${ }^{[12]}$. Roofs, parking lots ${ }^{[13]}$, lighting technologies ${ }^{[14]}$, and other urban features have all been mapped using hyperspectral imagery.

The second category is single-image super-resolution, which produces high-resolution HSI directly from low-resolution HSI. The traditional method interpolates the low resolution HSI using an interpolation method such as double tertiary interpolation to get the final estimation result, which is inferior. Due to the rapid development of deep learning in recent years, convolutional neural networks have shown excellent performance in natural image processing. Due to the similarity between natural and hyperspectral images, some scholars are introducing deep learning techniques into HSI super-resolution technology. For example, Li et al. ${ }^{[4]}$ proposed a deep recursive residual network to learn to map the input low resolution HSI directly to the high resolution HSI mapping network. Lietal. [5] also proposed a network structure. The structure learns the mapping relationship between the spectral differences between the low resolution HSI and the high resolution HSI. While these methods improve image resolution to some extent, they need to further improve the effectiveness of super-resolution due to the complex network structure of the application and limited performance.

In this research, we propose a hyperspectral image super-resolution method based on a deep residual convolutional neural network that can greatly enhance the super-resolution effect, and specifically divide it into two steps. The first step is to train the network through natural images and get a model that represents the mapping relationship between low and high resolution images. The second step is to combine transfer learning ideas, Applying the model to a hyperspectral image is to get a hyperspectral image at a high resolution rate.

\section{Proposal of research methods}

\subsection{Symbolization}

In this study, $D_{n}=\left\{\left(X_{n}, Y_{n}\right)\right\}$ represents low-resolution and high-resolution images of the natural image domain, and $D=\{X, Y\}$ represents the hyperspectral image domain image low-resolution 
hyperspectral image $Y \in R^{m \times n \times L}$ can be obtained by downsampling the original high resolution hyperspectral image $Y \in R^{M \times N \times L}$ by bicubic interpolation and reconstructing the high resolution. Image from the resulting X-resolution hyperspectral image $\mu^{\mu} \in R^{M \times N \times L}$. Among them, $\mathrm{M}, \mathrm{N}, \mathrm{m}, \mathrm{n}(\mathrm{M}>>\mathrm{m}, \mathrm{N}>>\mathrm{n})$ all represent the size of space, and $L$ represents the size. Of the spectrum.

\subsection{Deep residual convolutional neural network model}

With the powerful learning and representation capabilities of deep convolutional neural networks, this study uses a deep residual convolutional neural network (DRCNN) to capture the mapping between low-resolution and high-resolution image relationships. Remove unwanted stuff based on the DRCNN framework. In modules such as the batch standardization layer, a particular network structure consists of five main parts, including Conv1,ResBlock,Conv2,Upscale4x, and Conv3, as shown in Figure 1.

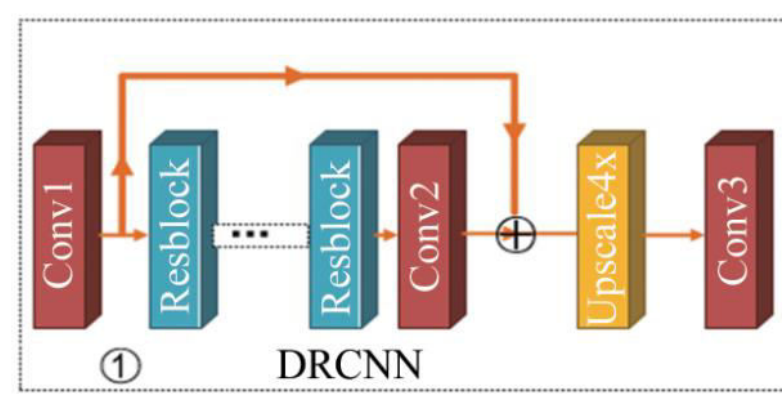

LR HSI

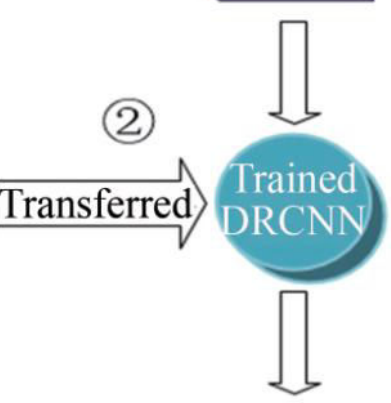

Estimated HR HSI

Fig.1 Proposed frame flow chart

It is agreed that $\operatorname{Conv}(c, f, k, s p)$ represents the convolutional layer. Among them:c is the number of input feature maps; $\mathrm{f}$ is the number of filters; $\mathrm{k}$ is the size of the convolution kernel; $\mathrm{s}$ is the step size; $\mathrm{p}$ is the number of padding. In the convolutional layer of the 5 parts of the network, specific values are assigned to these parameters respectively.

1)The first part contains the convolution layer Conv1(1,256,3,1,1) used to extract the initial features of the image, and the $3 \times 3$ convolution kernel convolves the input image. Perform the operation. Move the obtained result to the next layer.

2)The second part is the residual layer consisting of 32 residual blocks. Each residual block contains two convolutional layers and one activation layer, namely Conv $(256,256,3,1,1)$-ReLU- Conv $(256,256,3,1) ., 1)$. In addition, the residual block adds the input and output information as the final output result and outputs it to the next layer. 3) The third part contains the convolution layer Conv2 $(256,256,3,1,1)$ as the transition layer of the network, and the $3 \times 3$ convolution kernel performs the 
convolution operation on the output result of the residual layer. I will do it. At the same time, the result is added to the output result of the first part as the final output result and output to the next upscale $4 \mathrm{x}$ layer.

4)The fourth part is the upscale $4 x$ layer, which consists of two convolution layers and two Pixelshuffle layers, quadrupling the number of pixels in the feature map. That is, Conv $(256,256 \mathrm{x} 4,3$, 1, 1)-PixelShuffle (2)-Conv (256, $256 \times$ 4, 3, 1, 1)-PixelShuffle (2). The combination of the convolution layer and the pixel shuffle layer allows you to double the pixels in your image.

5)The fifth part contains a convolution layer Conv3 $(256,1,3,1,1)$ for integrating feature maps and outputting results, and the output results are considered super-resolution results.

\subsection{Model training}

Use the DIV2K dataset for training. The dataset contains 1000 natural images, some of which are taken. By superimposing and dicing these images at step size 8, a large number of image blocks are obtained. Is 80px x 80px x 3.The image in the RGB color space is then converted to the YCBCR color space and only the $\mathrm{Y}$ channel is selected,so the sample image used for training is a single channel.Next,the processed 80pxx80pxx1 image block is bicubic interpolated and downsampled to obtain an input image sample with a size of 20pxx20pxx1. At the same time, the labels corresponding to the image samples used as the original high resolution image block inputs, the input images and the corresponding labels form a training sample pair, and finally a training dataset of 109-200 sample pairs is formed and modeled. Used for training. In this paper, we use the luminance channel of a natural image for super-resolution, the Y channel in the YCBCR color space. The reason is that it uses a single channel natural image as input, so when the model is transferred to the hyperspectral image domain, the hyperspectral image looks like this: Entering a model band by band is equivalent to a single channel input for reconstructing an image.

DRCNN directly learns the mapping relationship between the low-resolution natural image $X_{n}$ and the high-resolution natural image $Y_{n}$. In order to facilitate the representation of the model, $F(\bullet)$ is used to represent the non-linear mapping relationship of the DRCCNN model. In addition, $F(\bullet)$ can be learned by minimizing the loss between the image $Y_{n}$ and the reconstructed image $F\left(X_{n}, \theta\right)$. Suppose the training data set is $D_{n}=\left\{\left(X_{n}^{(i)}, Y_{n}^{(i)}\right) \mid i=1,2, \cdots, N\right\}$.Among them: $X_{n}^{(i)}$ represents the i-th training sample, and its size is $20 \mathrm{p} \times \times 20 \mathrm{px} \times 1 ; Y_{n}^{(i)}$ represents the $\mathrm{i}$-th training label, and its size is $80 \mathrm{p} \times 80 \mathrm{p} \times \times 1 ; \mathrm{N}$ is the input image sample The total amount. Then the model can be trained by the following loss function:

$$
L(\theta)=\sum_{i=1}^{N} \mathrm{P} F\left(X_{n}^{(i)} ; \theta\right)-Y_{n}^{(i)} \mathrm{P}
$$

In the formula: $\theta=\left\{W_{(t)}, b_{(t)} \mid t=1,2, \cdots, T\right\}$ represents the parameters of the DRCNN model, and T represents the number of layers. In the model designed in this research, the L1Loss loss function is used to calculate the loss $L(\theta)$, which is equation (1); the Adam optimizer is used to update the parameter $\theta$. 


\subsection{Transfer the model to the hyperspectral image domain}

The low-resolution hyperspectral image generation model can be expressed as:

$$
X=Y \downarrow+n
$$

Where: $X \in R_{m \times n \times L}$ represents a low-resolution hyperspectral image, where $m \times n$ represents the number of pixels in each band, and $\mathrm{L}$ represents the number of spectral bands; $Y \in R_{M \times N \times L}$ represents a true hyperspectral image represents the down-sampling operation. The down-sampling operation is performed using the bicubic interpolation method, and the down-sampling factor is 4 .

Assuming that a low-resolution hyperspectral image $\mathrm{X}$ has been obtained, then it is necessary to transfer the previously trained DRCCNN model in the natural image domain to the hyperspectral image domain to obtain a high-resolution hyperspectral image $\mu^{\left[{ }^{[7]}\right.}$. Due to the strong generalization ability and representation ability of deep learning, this process can be implemented well, and the problem of insufficient samples of hyperspectral images for training can be solved well. As shown in the right half of Figure 1, the low-resolution hyperspectral $X \in R_{m \times x \times L}$ is brought into the trained model $F(\bullet)$ one by one to obtain a high-resolution hyperspectral image $\mu^{\mu} \in R^{M \times N \times L}$. The process of model transfer can be Simply expressed as:

$$
\boldsymbol{\mu}=\left[F\left(X_{1} ; \theta\right), F\left(X_{2} ; \theta\right), \cdots, F\left(X_{L} ; \theta\right)\right]
$$

Where: $X_{l} \in R^{m \times n}$ represents the image of the lth band of the low-resolution hyperspectral image X.

\section{Experimental test and analysis}

\subsection{Test database}

In order to effectively evaluate the performance of the proposed method, this study uses three databases including actual ground objects and remote sensing images. One is the CAVE database. This is a multispectral image database used to simulate GAP cameras and contains a variety of real-world materials and objects. It consists of 32 images with a size of 51 pixels x 512 pixels x 31 . Where 31 represents the number of spectral bands in the $400-700 \mathrm{~nm}$ range with a step size of $10 \mathrm{~nm}$. The second is the Pavia database. This database contains two scenes captured by ROSIS sensors during a flight battle over Pavia in northern Italy. One scene is the center of Pavia and the other is the University of Pavia. In this study, we selected the scene from the University of Pavia, and the image size is 610px $\mathrm{x}$ 340px x 103.Since some spectral bands in the image do not contain any information, the richer part of the original image is selected for testing and the final image size is 256 pixels $\mathrm{x} 256$ pixels $\mathrm{x} 93$. The third is the Washington Mall database. The scene is Washington Mall, each containing 191 spectral bands of 1280 pixels x 307 pixels. The image contains a great deal of object information and can be used to validate the processing associated with hyperspectral images.

\subsection{Experimental parameter settings and evaluation indicators}

This study uses the pytorch framework to implement the proposed network model. In the training 
phase, set the parameter batch_size to 16 , the learning rate $1 r$ to $10-4$, and $\mathrm{p}$ to 0.9 . In addition, L1Loss is used to calculate the loss and Adam Optimizer is used to update the parameter $\theta$ to minimize training loss.

In this study, six image quality metrics are used to comprehensively evaluate the reconstructed hyperspectral image. These are peak signal-to-noise ratio (PSNR), root mean square error (RMSE), spectral angle mapping (SAM), and relative dimensionless synthesis in synthesis. .. Overall error (ERGAS), Universal Quality Index (UIQI), and Structural Similarity (SSIM). From differences in pixel values between the reconstructed image and the original image, structural similarity, spectral distortion, and brightness distortion

All aspects are compared to achieve the goal of accurately measuring the quality of the reconstructed image.

\subsection{Experimental results}

Since it is not possible to display all the bands in the image, only three bands similar to the RGB bands in the reconstructed image are selected to build a pseudo-color image. For the Pavia database, select the 5th, 30th, and 55th bands to create a pseudo-color image. For the CAVE database, select the 5th,15th, and 25th bands to create a pseudo-color image. For the WashingtonMall database, select the 27th, 42nd, and 52nd bands to create a pseudo-color image.

Tables 1-3 compare the quality indicators of the proposed methods in three different databases with the other three competing methods. Figures 2-4 show a comparison of pseudo-color images of the proposed method in three different databases with the other three competing methods.

Tab.1 Comparison of evaluation indicators on the CAVE database

\begin{tabular}{ccccccc}
\hline \multirow{2}{*}{ method } & \multicolumn{7}{c}{ Fake_and_real_beers } \\
\cline { 2 - 7 } & PSNR & RMSE & SAM & ERGAS & UIQI & SSIM \\
\hline HySure & 28.2159 & 0.0388 & 5.5871 & 3.4048 & 0.9614 & 0.5967 \\
SDCNN & 32.4016 & 0.0240 & 2.1456 & 2.0786 & 0.9848 & 0.9406 \\
SRTL & 34.32228 & 0.0192 & 2.8989 & 1.7751 & 0.9899 & 0.9390 \\
Proposed & 39.7216 & 0.0103 & 0.8844 & 0.8962 & 0.9971 & 0.9797 \\
\hline
\end{tabular}

\begin{tabular}{ccccccc}
\hline \multirow{2}{*}{ method } & \multicolumn{7}{c}{ Fake_and_real_beers } \\
\cline { 2 - 7 } & PSNR & RMSE & SAM & ERGAS & UIQI & SSIM \\
\hline HySure & 34.1534 & 0.0196 & 19.4138 & 9.1201 & 0.9832 & 0.8310 \\
SDCNN & 32.5625 & 0.0235 & 9.3500 & 11.0226 & 0.9753 & 0.9433 \\
SRTL & 24.8046 & 0.0575 & 25.4859 & 29.1021 & 0.7978 & 0.5126 \\
Proposed & 41.5040 & 0.0084 & 2.9014 & 4.1945 & 0.9966 & 0.9872 \\
\hline
\end{tabular}

\begin{tabular}{ccccccc}
\hline \multirow{2}{*}{ method } & \multicolumn{7}{c|}{ Fake_and_real_beers } \\
\cline { 2 - 7 } & PSNR & RMSE & SAM & ERGAS & UIQI & SSIM \\
\hline HySure & 35.5390 & 0.0607 & 28.3861 & 7.5918 & 0.9838 & 0.8481 \\
SDCNN & 32.0095 & 0.0251 & 11.5548 & 11.4433 & 0.9645 & 0.9086 \\
SRTL & 24.3185 & 0.0608 & 18.0244 & 28.3238 & 0.7885 & 0.4386
\end{tabular}




\begin{tabular}{ccccccc} 
Proposed & 39.4984 & 0.0106 & 4.8519 & 4.8488 & 0.9935 & 0.9754 \\
\hline \multicolumn{7}{c}{ Tab.2 Comparison of evaluation indicators on the Pavia database } \\
method & PSNR & RMSE & SAM & ERGAS & UIQI & SSIM \\
HySure & 28.9095 & 0.0359 & 3.0014 & 3.1395 & 0.8784 & 0.6916 \\
SDCNN & 25.2173 & 0.0548 & 4.7988 & 4.9688 & 0.7572 & 0.6591 \\
SRTL & 30.7107 & 0.0291 & 2.6446 & 2.6452 & 0.9131 & 0.8265 \\
Proposed & 31.2558 & 0.0274 & 2.3343 & 2.4700 & 0.9240 & 0.8419 \\
\hline
\end{tabular}

Tab.3 Comparison of evaluation indicators on the Washington Mall database

\begin{tabular}{lcccccc}
\hline method & PSNR & RMSE & SAM & ERGAS & UIQI & \multicolumn{1}{c}{ SSIM } \\
\hline HySure & 24.9056 & 0.0568 & 1.9091 & 2.6741 & 0.1574 & 0.3495 \\
SDCNN & 36.0188 & 0.0158 & 1.0216 & 0.7092 & 0.6765 & 0.9323 \\
SRTL & 37.0930 & 0.0140 & 1.2353 & 0.6379 & 0.5186 & 0.9255 \\
Proposed & 40.3476 & 0.0096 & 0.6239 & 0.4357 & 0.6897 & 0.9638 \\
\hline
\end{tabular}

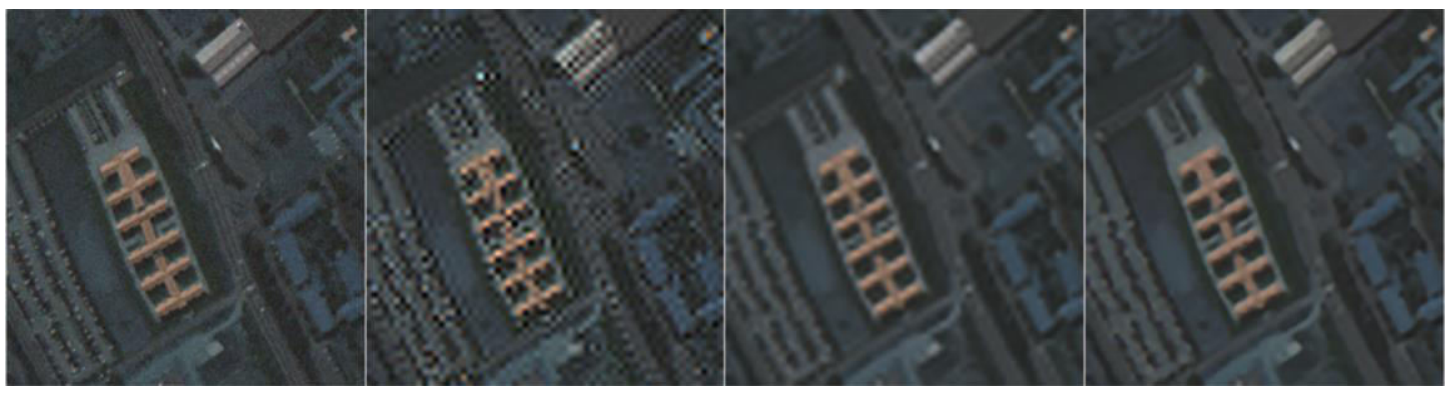
(a) HySure
(b) SDCNN
(c) SRTL
(d) Proposed

Fig.2 Comparison of pseudo - color images on the Pavia database by different methods

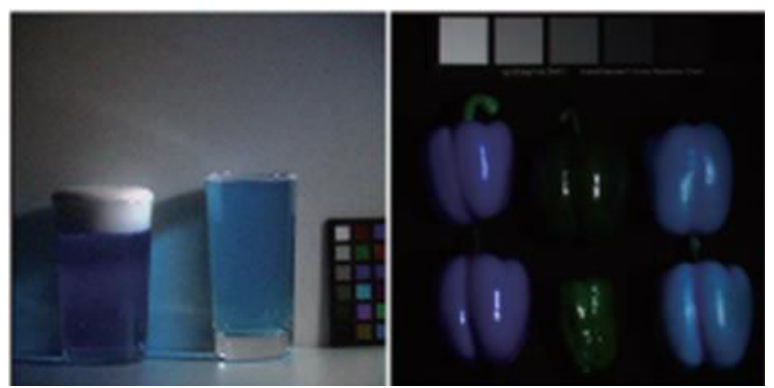

(a) HySure

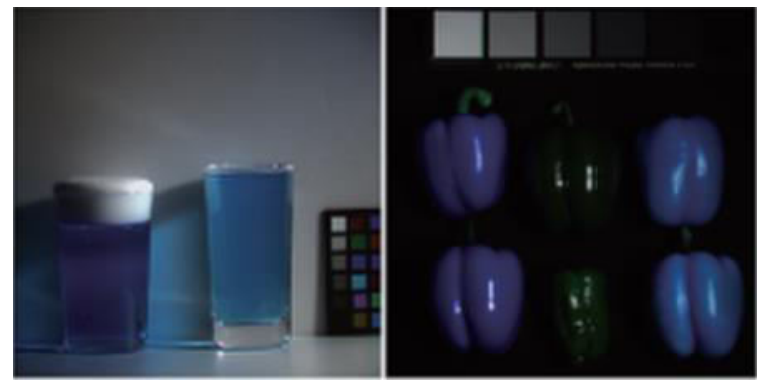


(b) SDCNN

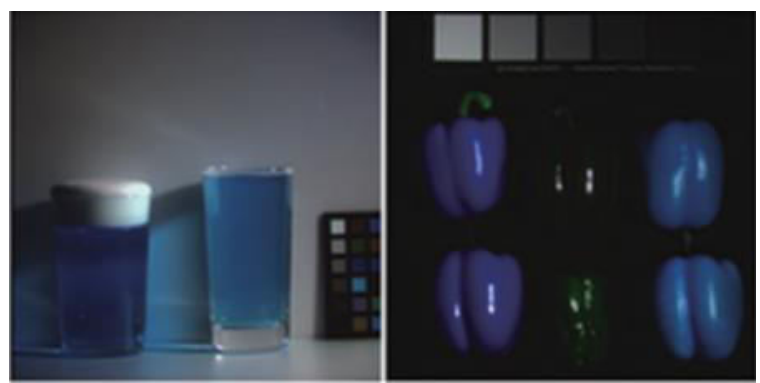

(c) SRTL

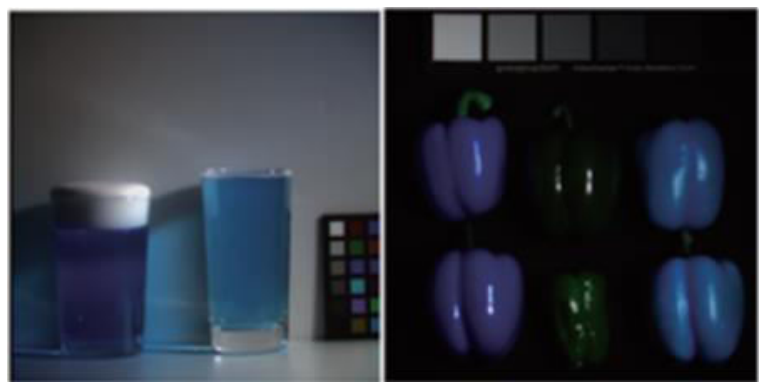

(d) Proposed

Fig.3 Comparison of pseudo - color images on the CAVE database by different methods

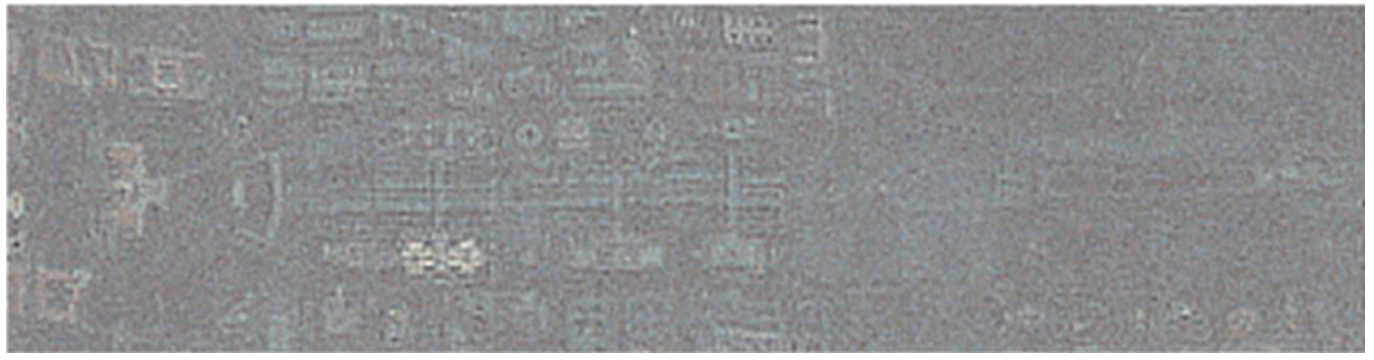

(a) HySure

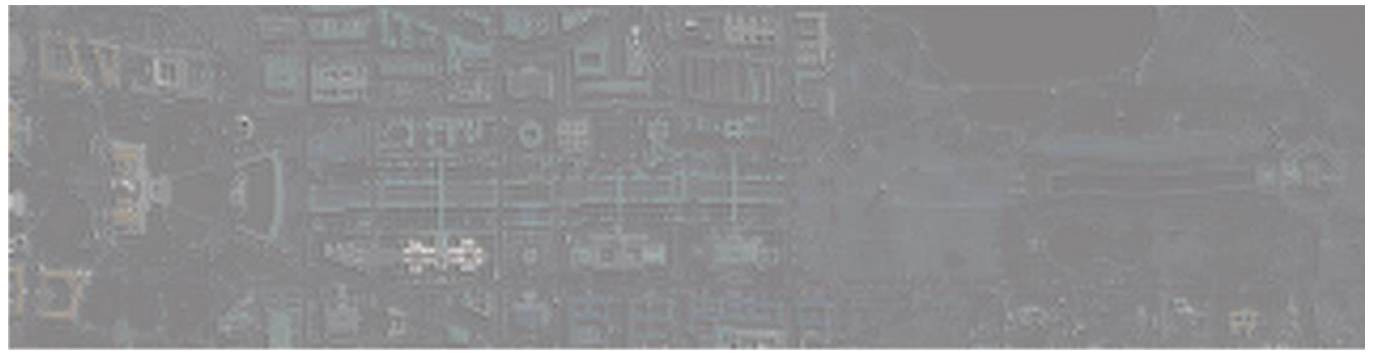

(b) SDCNN

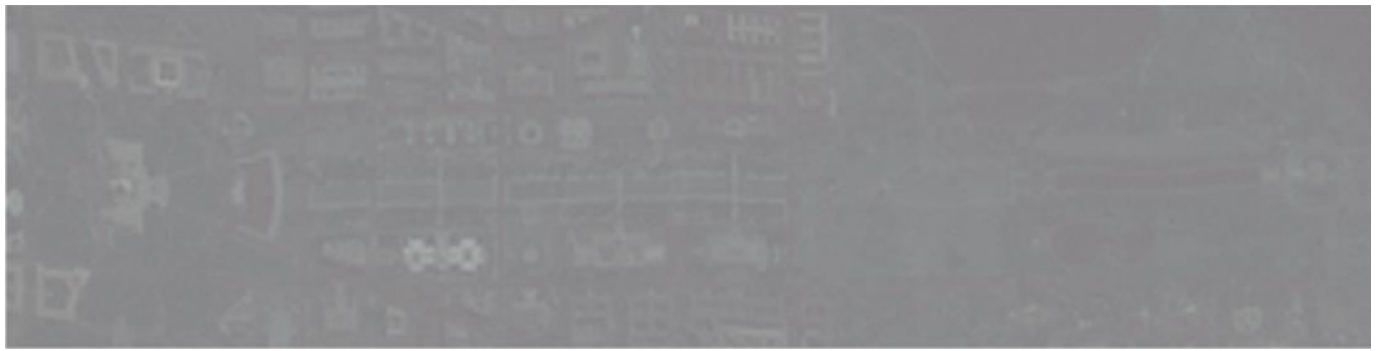

(c) SRTL 


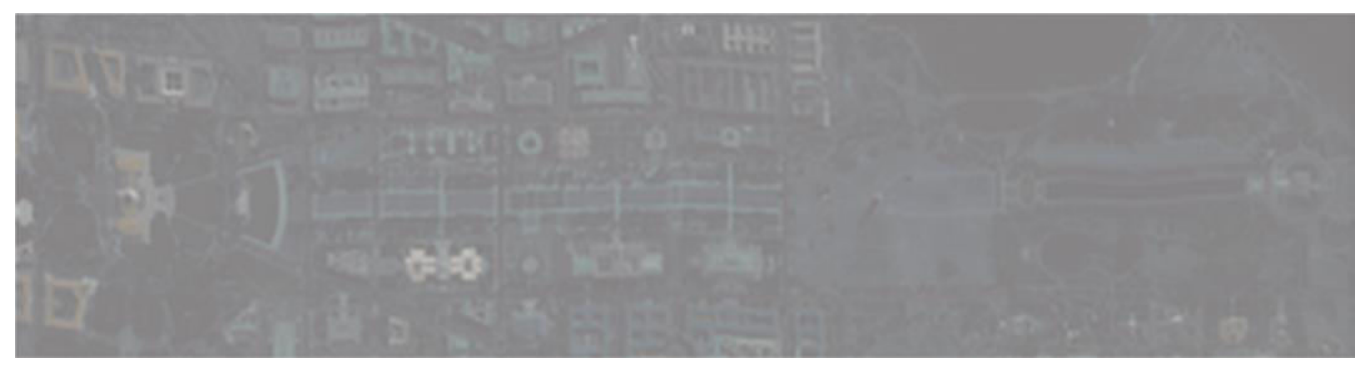

(d) Proposed

Fig.4 Comparison of pseudo - color images on the Washington Mall database by different methods

From the experimental results in Tables 1-3 and Figures 2-4, for hyperspectral databases with three different features, the method proposed in this study is super-resolution in terms of some quality metrics or visuals. I understand this. -The resolution effect of hyperspectral images is superior to the HySure, SDCNN and SRTL methods, and the effect has obvious advantages.

Specifically, in the CAVE database, the PSNR values of the method proposed in this study averaged 7.61 higher than the HySure method, 7.92 higher than the SDCNN method, and 12.43 higher than the SRTL method. As mentioned above, the PSNR value of this method is 2.35 higher than the HySure method, 6.04 higher than the SDCNN method, and 0.55 higher than the SRTL method. In the WashingtonMall database, the PSNR value for this method is higher than the HySure method 15.44. , This is 4.33 higher than the SDCNN method and 3.25 higher than the SRTL method. The methods proposed in this study show clear advantages over the three competing methods.

\section{Conclusions and Future Work}

Using numerous multi-spectral sensors with different filters, as demonstrated in this study, offers up a new technique to obtain hyperspectral data from a WSN. DCS produced the greatest results for calculating the high-resolution spectrum, but requiring less a priori information than UPDM. UPDM is extremely dependent on base spectra selection, making general use difficult; it is only suitable for small networks in homogeneous locations. Secondly, this study proposes a super-resolution method for hyperspectral images based on deep residual convolutional neural networks that reconstructs high-resolution hyperspectral images using only low-resolution hyperspectral images. The proposed method uses an improved deep residual convolutional neural network to significantly improve the resolution of the image. The model used reduces unnecessary modules such as batch standardization layers. Not only does this not affect the super-resolution effect, it also improves the efficiency of the model. At the same time, using the theory of transfer learning, the model is successfully applied to hyperspectral images, solving the problem of inadequate training samples of hyperspectral images and ultimately achieving better image reconstruction effects. You can get it. Through experiments with recognized real-world remote sensing datasets, the methods proposed in this study have been shown to be more. This method has better super-resolution performance. 


\section{Data Availability}

The datasets used during the current study are available from the corresponding author on reasonable request.

\section{Conflicts of Interest}

The author declares that there are no conflicts of interest.

\section{Acknowledgments}

I do not receive any funding.

\section{Consent}

I have full consent for the publication of this paper.

\section{References}

[1] AKHTAR N, SHAFAIT F, MIAN A. Bayesian sparse representation for hyperspectral image super resolution[C]//IEEE Conference on Computer Vision and Pattern Recognition. New York: IEEE2015: $3631-3640$.

[2] DONG W S, FU F Z, SHI G M, et al. Hyperspectral image super-resolution via nonnegative structured sparse representation [J]. IEEE Transactions on Image Processing, 2016, 25:2337 -2352 .

[3] SIMOES M,JOSE B D,ALMEIDA L B,et al.A convex formulation for hyperspectral image super resolution via subspace - based regularization [J]. IEEE Transactions on Geoscience and Remote Sensing, 2015,53:3373-3388.

[4] LI Y, ZHANG L, CHEN D L, et al. Single hyperspectral image super - resolution with grouped deep recursive residual network [C]//IEEE Fourth International Conference on Multimedia Big Data. New York: IEEE, 2018:1-4.

[5] LI Y S, HU J, ZHAO X, et al. Hyperspectral image super-resolution using deep convolutional neural network $[\mathrm{J}]$. Neurocomputing, 2017, 266:29-41.

[6] LIM B, SON S, KIM H, et al. Enhanced deep residual networks for single image super resolution[C]//IEEE Conference on Computer Vision and Pattern Recognition Workshops. New York IEEE， 2017:136-144.

[7] HU J, LU J, TAN Y P. Deep transfer metric learning[J ]. IEEE Conf Comput Vis Pattern Recognit, $2015,25: 325-333$.

[8] YUAN Y, ZHENG X T, LU X Q. Hyperspectral image super resolution by transfer learning [J]. IEEE Journal of Selected Topics in Applied Earth Observations and Remote Sensing, 2017, 10:1963-1974.

[9] NAN F, ZENG Q, XING Y, et al. Single image super resolution reconstruction based on the ResNeXt network [J]. Multimedia Tools and Applications, 2020, 79 (7) : 34459-34470.

[10] REN S, HE K, GIRSHICK R, et al. Faster R-CNN :towards real-time object detection with region proposal networks [J]. IEEE Transactions on Pattern Analysis and Machine Intelligence, 2017, 39 (6) : 1137-1149. 
[11] Dale, Laura M., et al. "Hyperspectral imaging applications in agriculture and agro-food product quality and safety control: A review." Applied Spectroscopy Reviews 48.2 (2013): 142-159.

[12] Van der Meer, Freek D., et al. "Multi-and hyperspectral geologic remote sensing: A review." International Journal of Applied Earth Observation and Geoinformation 14.1 (2012): 112-128.

[13] Dell'Acqua, Fabio, et al. "Exploiting spectral and spatial information in hyperspectral urban data with high resolution." IEEE Geoscience and Remote Sensing Letters 1.4 (2004): 322-326.

[14] Dobler, Gregory, et al. "A hyperspectral survey of New York City lighting technology." Sensors 16.12 (2016): 2047. 